\title{
IL6/STAT Signaling Pathway and PPARa are Involved in Mediating the dose Dependent Cardioprotective Effect of Fenofibrate in 5- fluorouracil induced Cardiotoxicity
}

marwa refaie ( $\sim$ marwamonier@yahoo.com )

Minia University Faculty of Medicine

Sayed Shehata

Minia University Faculty of Medicine

Asmaa M.A. Bayoumi

minia university

Nashwa Fathy Gamal El-Tahawy

minia university

Walaa Y. Abdelzaher

minia university

\section{Research Article}

Keywords: 5-Fluorouracil, Fenofibrate, Cardiotoxicity, Interleukin 6

Posted Date: April 16th, 2021

DOl: https://doi.org/10.21203/rs.3.rs-415786/v1

License: (c) (1) This work is licensed under a Creative Commons Attribution 4.0 International License.

Read Full License 


\section{Abstract}

Purpose: Cardiotoxicity of anticancer drugs such as 5-fluorouracil (5FU) and anthracyclines is a major complication that challenges their clinical usefulness. There is a critical need for finding new protecting drugs to defense against these harmful side effects. Up till now, there are no studies evaluate the possible cardioprotective effects of fenofibrate (FEN) in 5FU-induced cardiotoxicity. So that, we aimed in current model to evaluate such effect of FEN and explore different mechanisms mediating it.

Methods: we used FEN $(25,50,100 \mathrm{mg} / \mathrm{kg} /$ day $)$ orally for 7days with induction of cardiotoxicity by intraperitoneal (i.p.) injection of $5 \mathrm{FU}(150 \mathrm{mg} / \mathrm{kg})$ on $5^{\text {th }}$ day.

Results: current study showed that 5FU succeeded in induction of cardiotoxicity and manifested by significant elevation of cardiac enzymes, tissue malondialdehyde (MDA), interleukin 6 (IL6), signal transducer and activator of transcription 4(STAT4) and caspase 3 levels. Furthermore, 5FU group showed toxic histopathological changes of marked cardiac damage and significant decrease in reduced glutathione (GSH), total antioxidant capacity (TAC) and peroxisome proliferator activated receptor alpha (PPARa) expression. FEN could reverse 5FU-induced cardiotoxicity by different mechanisms including upregulation of PPARa, inhibition of IL6/STAT signaling pathway, anti-inflammatory, anti-apoptotic and anti-oxidant properties.

Conclusion: FEN had a significant cardioprotective effect against 5FU induced cardiac damage.

\section{Introduction}

5-Fluorouracil (5FU) is a cytotoxic thymidylate synthase inhibitor and it is widely used for the treatment of different malignancies. However, its use is associated with serious cardiotoxic effects including cardiac arrhythmias, myocardial ischemia, left ventricular dysfunction, hyper and hypotension, cardiac arrest, and sudden death ${ }^{1,2}$. $5 \mathrm{FU}$ induces oxidative stress with massive release of reactive oxygen species (ROS) but the heart exhibits relatively low level of antioxidant enzymes. For example, cardiac tissue contains 4 times less of superoxide dismutase (SOD) and 150 times less of catalase than liver ${ }^{3}$.

Early response to myocardial injury and inflammatory signaling in cardiomyocytes entails the activation of nuclear factor-kB with increased expression of proinflammatory cytokines such as tumor necrosis factor-alpha and interleukin-6 (IL6) followed by activation of Janus kinase/signal transducer and activator of transcription (JAK/STAT) signaling pathway. It is an essential intracellular signaling pathway and closely related to cardiac hypertrophy. This pathway has a key role in cell survival, growth, and differentiation ${ }^{4-6}$.

Upon phosphorylation of JAK/STAT, it translocates inside the nucleus, binds with the promoter region of the target genes, and regulates their transcription. In the cardiac tissue, STATs regulate the expression of gene encoding proteins involved in extracellular matrix composition, angiogenesis, inflammation and apoptosis. IL-6 and the family-related proteins play a critical role in development of cardiac hypertrophy 
and heart failure. Therefore, modulation of the IL6 /STAT signaling pathway represents an essential strategy for the treatment of 5FU-induced cardiac injury ${ }^{5,7,8}$.

Peroxisome proliferator-activated receptors (PPARs) are nuclear receptors. It includes three isotypes encoded by different genes: PPAR $\alpha$, PPAR $\beta / \delta$ and PPAR $\gamma$. They are involved in fatty acid, lipoprotein metabolism, immune response, glucose homeostasis, cellular proliferation and differentiation. Accordingly, PPARs are important targets in modulation of different disorders ${ }^{9}$.

PPARa activation leads to increase the expression of various antioxidant enzymes which results in elevating the redox state and represses inflammation and apoptosis. Its activation leads to increase gene expression of $\mathrm{Cu} / \mathrm{Zn}$ SOD, glutathione reductase, and glutathione-S-transferase. Moreover, PPARa represses nuclear factor kappa $b$ and activator protein- 1 thereby down regulates the inflammatory response and oxidative stress. However, PPARa deficiency is associated with disordered fatty acid metabolism accompanied with insulin resistance ${ }^{10}$.

Fenofibrate (FEN) is one of fibrate family which is PPARa receptor agonists. It is a specific ligand of PPARa that could bind to and specifically activate the transcription of these receptors. It is commonly used for the treatment of hyperlipidemia. Recently, different animal models have demonstrated that FEN could attenuate different tissue injuries depending on its pleotropic effects. It has the ability to upregulate PPARa receptor, anti-oxidant, anti-inflammatory and anti-apoptotic properties ${ }^{1,10}$.

Increasing life expectancy of cancer patients and saving different organs against 5FU harmful effects are still in critical need. This requirement and different mechanisms of FEN forced us to investigate the possible cardioprotective effect of FEN in 5FU- induced cardiac damage and study the mechanisms involved in such protection including IL6/STAT pathway and the role of PPARa receptors.

\section{Materials And Methods}

\subsection{Ethics}

Animal handling, medications, and scarification were carried out following the guidelines for the care of experimental animals and approved by the Institutional Ethical Committee, Faculty of Medicine, Minia University, Egypt according to the NIH Guide for taking care and use of laboratory animals. Approval No. 19:3/2021

\subsection{Chemicals}

5FU was bought from Sigma-Aldrich Co. (St. Louis, MO, USA) and FEN was obtained from Mina Pharm Company, Egypt. Total antioxidant capacity (TAC) and reduced glutathione (GSH) kits were purchased from Biodiagnostic, Egypt. Alanine transaminase (ALT), aspartate transaminase (AST), lactate dehydrogenase (LDH), and creatinine kits were from BioMed, Egypt. Creatinine kinase-MB (CK-MB) was purchased using commercial kits Spectrum Diagnostic, Egypt. Rabbit polyclonal anti-IL6 and anti-cleaved 
caspase 3 antibodies were from Neo Markers Co., CA. Mouse monoclonal anti- $\beta$-actin antibody and secondary antibodies were from Sigma-Aldrich Co., USA.

\subsection{Animals and experimental design}

Forty male Wistar albino rats weighing 180-210 g were obtained from the National Center of Research, El-Giza, Egypt. After two weeks of acclimatization to the environmental conditions (12 h lighting cycle, 25 $\pm 2^{\circ} \mathrm{C}$ temperature, $45 \pm 5 \%$ humidity, and free access to water and standard chow from El-Nile Company, Egypt), the rodents were assorted into 5 groups (8 rats /group).

\section{Group I (control) (CON)}

received carboxymethyl cellulose $(1 \% \mathrm{CMC}, 1 \mathrm{ml} / \mathrm{rat})$ daily by oral gavage as a vehicle for 7 days.

\section{Group II (5FU)}

received 5FU (150 mg $\backslash \mathrm{kg}$ i.p. injection on the 5 th day ${ }^{11,12}$.

\section{Group III (FEN25)}

treated with 5FU (150 mg $\backslash \mathrm{kg}$ i.p injection on the 5 th day) ${ }^{11,12}$ plus FEN (25mg/kg/day) ${ }^{13}$ orally for 7 days.

\section{Group IV (FEN50)}

treated with $5 F U$ (150 mg $\backslash \mathrm{kg}$ i.p injection on the 5 th day) ${ }^{11,12}$ and FEN (50mg/kg/day) ${ }^{13,14}$ orally for 7 days.

\section{Group V (FEN100)}

treated with 5FU (150 mg $\backslash \mathrm{kg}$ i.p injection on the 5 th day) ${ }^{11,12}$ and FEN (100mg/kg/day) ${ }^{10,13,14}$ orally for 7days.

\subsection{Blood and tissue sampling}

To terminate the experiment, the rodents were sacrificed. They were anesthetized with i.p. injection of urethane $(20 \%$ in a dose of $1 \mathrm{gm} / \mathrm{kg})$. Blood was obtained from the abdominal aorta, left to coagulate at room temperature, centrifuged at 5000 rpm for 15 minutes (JanetzkiT30 centrifuge, Germany), and then sera were frozen at $-80^{\circ} \mathrm{C}$ for further analysis. The hearts were excised, washed from the blood by saline, and divided. Samples from the left ventricle were kept in 10\% formalin for histopathological and immunohistochemical studies. Other cardiac specimens were homogenized in ice-cold phosphate buffer $(0.01 \mathrm{M}, \mathrm{pH} 7.4 ; 20 \% \mathrm{w} / \mathrm{v})$, centrifuged for 15 minutes at $5000 \mathrm{rpm}$, and the supernatant was frozen at $-80^{\circ} \mathrm{C}$ for further biochemical examination.

\subsection{Biochemical analysis}




\subsubsection{Quantification of oxidative stress parameters in cardiac tissue}

Malondialdehyde (MDA) level, the index of lipid peroxidation, was determined following the method of Buege and Aust ${ }^{15}$. TAC and GSH were measured using colorimetric kits and following the manufacturer's instructions.

\subsubsection{Determination of cardiac biomarkers}

The serum levels of CK-MB, LDH, ALT and AST were quantified using commercial kits and following the manufacturer's instructions.

\subsection{Histological procedures}

Myocardial tissues were fixed in $10 \%$ formalin solution and processed for preparation of paraffin blocks. Six-micrometer sections were deparaffinized with xylene, rehydrated and used for hematoxylin and eosin (H\&E) staining using standard techniques ${ }^{16}$. The histopathological changes were semi-quantitatively scored according to the following degrees of histological myocardial injury: (0) No change: (1) Mild small multifocal damage with slight inflammation, (2) Moderate - extensive damage and/or diffuse inflammatory infiltration, (3) Severe - damage with necrosis and diffuse inflammatory process ${ }^{17}$.

Other deparaffinized and rehydrated sections were immunostained STAT4 using Anti-STAT4 antibody produced in rabbit (MFCD00803568, Sigma-Aldrich, Egypt) according to the manufacture's guidelines. Briefly, sections were incubated with trypsin then washed with PBS. The nonspecific binding sites were blocked in normal goat serum (1:50), incubated for $30 \mathrm{~min}$ with the primary STAT4 (1:200). Then washed with PBS and incubated with the secondary antibody (Vector laboratory 1:2000). Sites of peroxidase binding were detected using chromogenic 3,3'-diaminobenzidine (DAB) tetra hydrochloride substrate. Tissue sections were lastly counterstained with hematoxylin ${ }^{18}$.

\subsection{Image capture and morphometric analysis}

Sections were examined using a light microscope (Olympus, Tokyo, Japan). Images were digitally captured by a digital camera (ToupView, Zhejiang, China) using the ToupView software (version $\times 36$, 3.5.563; Hangzhou ToupTek Photonics Co., Zhejiang, China). Number of STAT4 immune-positive cells was counted in 3 non overlapping sections for 8 rats in each group.

\section{8. rt-PCR for the relative quantification of PPARa gene expression changes}

Total RNA was extracted using the ribozol RNA extraction reagent (Amresco, Solon, USA) according to the manufacturer's instructions. cDNAs were developed by the Revert Aid ${ }^{\text {TM }}$ First Strand cDNA Synthesis kit (Fermentas, Life Sciences). cDNA was reversely transcribed from $5 \mu \mathrm{g}$ of RNA in a thermal cycler at $42^{\circ} \mathrm{C}$ for $60 \mathrm{~min}$ then $70^{\circ} \mathrm{C}$ for $5 \mathrm{~min}$ according to the kit protocol. rt-PCR was performed with $50 \mathrm{ng}$ cDNA per reaction using $25 \mu \mathrm{L}$ of SYBR Green QPCR Mix (Solis BioDyne) containing $20 \mu \mathrm{M}$ of specific primers in the 
rt-PCR Detection System. The SYBR green data was evaluated by quantification of GAPDH (Glyceraldehyde-3-phosphate dehydrogenase) as reference gene ${ }^{19}$. The sets of primers used were as follows:

\section{GAPDH primers}

Forward: 5' GTCGGTGTGAACGGATTTG3'

Reverse: 5' CTTGCCGTGGGTAGAGTCAT3'

\section{PPAR a primers}

Sense: 5'ACGATGCTGTCCTCCTTGATG3'

Antisense: 5' GCGTCTGACTCGGTCTTCTT3'

\subsection{Western blotting}

For estimation of tissue expression of IL6 and cleaved caspase3, western blotting was performed as previously described (Ewees et al., 2018). Briefly, cardiac tissue was homogenized and then the homogenate was applied to sodium dodecyl sulphate-polyacrylamide gel electrophoresis (SDS-PAGE). Protein bands were transferred to a nitrocellulose membrane using a semi-dry blotter (Bio-Rad). The blot was blocked by TBS-T buffer containing 5\% skim milk powder for $1 \mathrm{~h}$ at room temperature. Then, the blot was probed overnight at $4^{\circ} \mathrm{C}$ with a rabbit polyclonal anti-IL6, anti-cleaved caspase3 or mouse monoclonal anti- $\beta$-actin antibodies. Subsequently, incubation with alkaline phosphatase-coupled secondary antibody (Sigma-Aldrich Co., USA) was performed for $1 \mathrm{~h}$ at room temperature. Blots were analyzed using BCIP/NBT (5-bromo-4-chloro-3'-indolyphosphate and nitro-blue tetrazolium) colorimetric method (Sigma-Aldrich Co., USA). Protein bands on the blots were analyzed using Image-J and GraphPad Prism-5 softwares.

\subsection{Statistical Analysis.}

All results were demonstrated as mean \pm standard error of the mean (SEM). One-way analysis of variance (ANOVA) and the Tukey-Kramer post-analysis test were performed to analyze the values. $P$-value of less than 0.05 was assigned for significance. GraphPad Prism was used for the statistical analysis (version 5.01 for Windows, Graph pad Software, San Diego California USA).

All data is available in prism files on requirement

\section{Results}

\subsection{Effect of FEN in different doses on oxidative stress parameters on 5FU- induced cardiotoxicity in rats}


In Table 1, 5FU group showed a significant increase in cardiac MDA with significant decrease in cardiac GSH and TAC when compared to control group. FEN in different doses, significantly improved oxidative stress parameters when compared to 5FU group. Meanwhile, coadministration of FEN100 mg/kg/day showed more improvement than $25,50 \mathrm{mg} / \mathrm{kg} /$ day.

Table 1

Effect of FEN in different doses on oxidative stress parameters in 5FU induced cardiotoxicity in rats

\begin{tabular}{|c|c|c|c|}
\hline Groups & $\begin{array}{l}\text { MDA } \\
\text { (nmol/g tissue) }\end{array}$ & $\begin{array}{l}\text { GSH } \\
\text { ( } \mu \mathrm{mol} / \mathrm{g} \text { tissue) }\end{array}$ & $\begin{array}{l}\text { TAC } \\
(\mathrm{mmol} / \mathrm{L})\end{array}$ \\
\hline Control & $182.5 \pm 5.041$ & $0.484 \pm 0.005$ & $0.384 \pm 0.010$ \\
\hline $5 F U$ & $712.0 \pm 14.37^{a}$ & $0.264 \pm 0.018^{a}$ & $0.120 \pm 0.006^{a}$ \\
\hline FEN25 & $428.2 \pm 31.49^{a b c}$ & $0.333 \pm 0.010^{\mathrm{abc}}$ & $0.211 \pm 0.008^{\mathrm{abc}}$ \\
\hline FEN50 & $294.6 \pm 12.61^{a b c}$ & $0.344 \pm 0.007^{a b c}$ & $0.305 \pm 0.009^{a b c}$ \\
\hline FEN100 & $195.6 \pm 5.681^{b}$ & $0.429 \pm 0.024^{b}$ & $0.381 \pm 0.009^{b}$ \\
\hline \multicolumn{4}{|c|}{$\begin{array}{l}\text { Results represent the mean } \pm \text { SEM }(n=8) .{ }^{\text {a }} \text { Significant }(P<0.05) \text { difference from control group. }{ }^{b} \\
\text { Significant }(P<0.05) \text { difference from } 5 F U \text { group. }{ }^{c} \text { Significant }(P<0.05) \text { difference from high dose } \\
\text { fenofibrate }(100 \mathrm{mg} / \mathrm{kg} / \text { day }) \text {. [ }[5 \mathrm{FU}=5 \text {-flurouracil; } F E N=\text { fenofibrate: MDA }=\text { malondialdehyde; } G S H= \\
\text { reduced glutathione: TAC = total antioxidant capacity }] .\end{array}$} \\
\hline
\end{tabular}

5FU group significantly increased levels of serum CK-MB, LDH, ALT and AST when compared to control group. FEN in different doses, significantly improved cardiac biomarkers when compared to $5 \mathrm{FU}$ group. On the other hand, FEN100 mg/kg/day plus 5FU showed more improvement than $25,50 \mathrm{mg} / \mathrm{kg} /$ day (Table 2). 
Table 2

Effect of FEN in different doses on cardiac biomarkers in 5FU induced cardiotoxicity in rats

\begin{tabular}{|lllll|}
\hline Groups & $\begin{array}{l}\text { CK-MB } \\
(\mathrm{U} / \mathrm{L})\end{array}$ & $\begin{array}{l}\text { LDH } \\
(\mathrm{U} / \mathrm{L})\end{array}$ & $\begin{array}{l}\text { ALT } \\
(\mathrm{U} / \mathrm{L})\end{array}$ & $\begin{array}{l}\text { AST } \\
(\mathrm{U} / \mathrm{L})\end{array}$ \\
\hline Control & $21.34 \pm 1.61$ & $73.36 \pm 2.89$ & $24.20 \pm 1.84$ & $30.63 \pm 2.16$ \\
\hline 5 -FU & $64.92 \pm 3.05^{\mathrm{a}}$ & $190.80 \pm 6.47^{\mathrm{a}}$ & $54.30 \pm 2.13^{\mathrm{a}}$ & $93.92 \pm 3.11^{\mathrm{a}}$ \\
\hline FEN25 & $45.07 \pm 2.81^{\mathrm{abc}}$ & $132.00 \pm 2.00^{\mathrm{abc}}$ & $39.95 \pm 2.03^{\mathrm{abc}}$ & $58.70 \pm 2.63^{\mathrm{abc}}$ \\
\hline FEN50 & $37.37 \pm 2.11^{\mathrm{abc}}$ & $111.20 \pm 4.66^{\mathrm{abc}}$ & $38.29 \pm 2.75^{\mathrm{abc}}$ & $48.52 \pm 1.72^{\mathrm{abc}}$ \\
\hline FEN100 & $23.45 \pm 1.44^{\mathrm{b}}$ & $84.49 \pm 5.20^{\mathrm{b}}$ & $27.36 \pm 2.52^{\mathrm{b}}$ & $34.54 \pm 1.93^{\mathrm{abc}}$ \\
\hline
\end{tabular}

Results represent the mean \pm SEM $(n=8)$. ${ }^{a}$ Significant $(P<0.05)$ difference from control group. ${ }^{b}$ Significant $(P<0.05)$ difference from $5 F U$ group. ${ }^{c}$ Significant $(P<0.05)$ difference from high dose fenofibrate $(100 \mathrm{mg} / \mathrm{kg} /$ day). [5FU = 5-flurouracil; FEN = fenofibrate; CK-MB: creatinine kinase-MB; LDH: lactate dehydrogenase; ALT: alanine transaminase; AST: aspartate transaminase].

\subsection{Histopathological evaluation of cardiac tissue}

Longitudinal sections of the control group showed normal cardiomyocytes with acidophilic cytoplasm, prominent striations and a single centrally located nucleus which showed branching. The myocytes were invested by connective tissue stroma. Fibroblast nuclei were found within the connective tissue or at the periphery of a muscle fiber. Many capillaries were seen among the cardiac muscle fibers. While the 5FU group showed cardiac muscle fiber injury in the form of damaged myofibrils (hyper-eosinophilic fibers with pyknotic nuclei and loss of striation), interstitial oedema, marked capillary congestion and inflammatory cells infiltration. Rats from FEN25, FEN50 and FEN100-treated groups revealed an obvious decrease in the histopathological changes occurred in the 5FU group. Notably, the greatest improvement appeared in FEN100-treated group (Fig. 1).

\subsection{Grading the histopathological findings of cardiac tissue}

Cardiac injury in the 5FU group showed a significant difference regarding the degenerated muscle fibers, inflammatory cells infiltration and vascular congestion compared to the control group. Rats from FEN25treated group, FEN50-treated group and FEN100-treated group had significant decreases in these changes compared to 5 -FU group (all $p<0.0001$ ). It was of interest to observe the marked decrease in FEN100treated group with a significant difference compared to both FEN25-treated group and FEN50-treated group (all $p<0.05$ ).

\subsection{Evaluation of STAT4 immunoexpression}

The morphometric studies and statistical analysis of the mean number of STAT4 immunopositive cells showed that there was significant increase in STAT4 immunostaining in 5FU group compared to the control group. However, treatment with FEN in its different doses showed a significant decrease in 
immunostaining compared to 5FU group. Interestingly, there was marked decrease in FEN 100 group (Fig. 2).

\subsection{Gene expression changes of PPARa}

There is a significant decrease of PPARa gene expression in 5FU given group in comparison with control group. However; there is a significant increase of its gene expression in 5FU + FEN given groups in comparison with 5FU given group alone (Fig. 3). Interestingly, FEN100 group showed more improvement than that found with FEN25,50 groups.

\subsection{Detection of IL6 and cleaved caspase 3 levels in cardiac tissue using western blotting}

There are significant increases of IL6 and cleaved caspase3 levels in 5FU given group in comparison with control group. However; coadministration of FEN showed significant decrease of cleaved caspase 3 and IL6 levels in comparison with 5FU given group alone (Fig. 4,5).

\section{Discussion}

5FU is a pyrimidine anti-metabolite and widely used for various malignant disorders including gastrointestinal, breast, neck, head, colon, and pancreatic cancers. Despite its vast therapeutic uses, it is well known to have severe cardiotoxic effects. Searching for new protective therapies to reduce these harmful effects is highly required ${ }^{21}$.

We aimed in our study to reduce 5FU induced cardiotoxicity using FEN and study the different mechanisms involved in such protection. $5 \mathrm{FU}$ given group showed marked increases in serum LDH, CKMB, ALT, AST, MDA, IL6, STAT, caspase3 level with marked tissue damage as shown in the histopathological features. However, there is significant decrease in GSH, TAC, PPARa expression. Interestingly, the current findings showed that FEN coadministration with 5FU had marked cardioprotective effect mediated mainly via upregulation of PPARa, ROS scavenging, suppression of inflammation, oxidative stress, apoptosis and modulation of IL6/STAT pathway.

Myocardium contains high concentrations of diagnostic markers released into the extracellular fluid during cardiac damage such as serum CK-MB, LDH, ALT and AST. Assay of the CK-MB isoenzyme activity is the most specific indicator for cardiac injury in serum. The increased activity of serum CK-MB isoenzyme reflects the alterations in the plasma membrane integrity and permeability ${ }^{12}$.

In the present study, 5FU treated rats showed a significant elevation in the activity of serum CK-MB, LDH, ALT, AST levels indicating 5FU induced myocardial necrotic damage and the leakiness of the plasma membrane. This is in accordance with Lamberti et al ${ }^{1}$; Desai et al ${ }^{22}$.

Different mechanisms of cardiotoxicity are proposed, including direct toxic action on myocytes, coronary vasospasm and thrombogenic effects. Moreover, 5FU induces endothelial damage, inflammatory reaction 
and myofibril necrosis. Impairment of Krebs' cycle and energy production within the myocytes is another theory of $5 \mathrm{FU}$ induced damage. Emerging evidence indicated the marked involvement of oxidative stress in the pathogenesis of 5FU-induced cardiac injury via excessive release of free radicals and ROS ${ }^{12}$.

5-FU-induced cytotoxicity is linked to the enhanced ROS formation and superoxide anion $\left(\mathrm{O}^{-2}\right)$ production. As a consequence, there is triggering of apoptotic program and cardiomyocyte damage. These ROS attack any molecules, but their main target is the polyenoic fatty acids within cell membranes forming peroxyl radicals causing a chain of reactions of membrane lipid peroxidation and myocardial necrosis. MDA is used for detection of membrane lipid peroxidation. It was elevated in the hearts of guinea pig ${ }^{23}$ and rat ${ }^{24}$ after 5FU-treatment. The is in agreement with our results that showed significant increase of MDA in addition to depletion of GSH and TAC due to marked increase in oxidative stress and excessive release of ROS. These findings are in line with other previous reports ${ }^{12,25}$.

Increased IL6 signaling in the myocardium mediates cardiac hypertrophy, decreases contractile function, heart failure and diabetic cardiomyopathy. Interestingly, the IL6-driven decrease in cardiac function is associated with the JAK/STAT signaling. This pathway triggers multiple signals involved in homeostasis and inflammation. The JAK-STAT signaling also plays a central role in transducing stress and growth signals in the cardiac disorders. JAK and STAT proteins are activated through ligand binding, after that phosphorylation and formation of the homo or heterodimers occurred. They translocate into the nucleus where their ability to regulate gene expression ${ }^{26}$.

In mammals, the JAK/STAT pathway transduces signals for a wide variety of cytokines and growth factors such as IL-1 $\beta$, Angll, TNF- $a$, IL- 6 and IFN- $\gamma$, all of which are involved in cardiac disorders.

Therefore; regulation of IL6/STAT pathway is so important in controlling 5FU induced cardiotoxicity and an essential target to prevent such damage and preserve the cardiac tissue. The same was found in our model that showed increased IL6/STAT pathway expression in 5FU given group. However, coadministration of FEN had significant decrease in IL6/STAT pathway expression compared to $5 \mathrm{FU}$ given group alone. These results are supported by other studies $2,5,26$.

Proinflammatory cytokines e.g., TNF- $a$ and IL6 are responsible for stimulation of the inflammatory/apoptotic pathways including caspase mediated apoptosis. Several caspases are highly involved in the early stages of apoptosis. Caspase3 is one member of this family and it is responsible for the initiation of the apoptotic process. It is activated by upstream caspase- 8 and caspase- 927,28 . The current findings demonstrated that 5FU could induce proinflammatory cytokines as IL6/STAT signaling pathway followed by activation of the apoptotic pathway as confirmed by increased caspase3, the reliable indices of apoptosis. The same was found with Lamberti et al ${ }^{1}$; Arab et al ${ }^{29}$; Muhammad et al 30 .

We found that there is marked improvement and normalization of 5FU-induced cardiac injury on coadministration of FEN. There are significant decreases in serum CK-MB, LDH, ALT, AST, MDA, caspase3, IL6, STAT. However, there are significant increases in TAC, GSH, PPARa gene expression. 
These results were further supported by the histopathological findings which exhibited normal structure with mild inflammatory cell infiltration in FEN given group. This is in line with Krysiak et al ${ }^{31}$; Jen et al ${ }^{32}$; Khan et al ${ }^{33}$. Protective effect of FEN may be attributed to its pleotropic effects in form of upregulation of PPARa, anti-oxidant, ant-inflammatory and anti-apoptotic effects and inhibition of one of the most critical pathways IL6/STAT pathway that is highly involved in mediating 5FU induced cardiac damage.

This is in line with Khan et al ${ }^{33}$; Wang et al ${ }^{34}$; Huang et al ${ }^{35}$. Further studies are highly recommended to examine FEN cardiopreserving properties in patients receiving $5 F U$.

\section{Conclusion}

Fenofibrate markedly protected against 5-fluorouracil -induced cardiotoxicity in dose dependent manner most probably due to PPARa upregulation, anti-oxidant, anti-apoptotic and anti-inflammatory activities with modulation of IL6/STAT pathway. Further studies are in need to evaluate its protective effect in human receiving 5 -fluorouracil chemotherapy.

\section{Declarations}

\section{Authors' contribution}

Dr. Marwa, Dr.Walaa and Dr. Sayed selected the point, performed the experimental part, wrote the manuscript and sent it for publication. Dr. Nashwa performed and wrote the histopathology, immunohistochemistry and revised the manuscript. Dr. Asmaa performed and wrote the part of western blotting and revised the manuscript.

\section{Acknowledgement}

All our thanks and appreciation for Dr. Wedad M. Abdelraheem, Department of Medical Microbiology and Immunology, Faculty of Medicine, Minia University, El-Minia, Egypt for helping us in rt-PCR part.

\section{Conflict of Interest}

The authors declare that they have no conflict of interest.

This research did not receive any specific grant from funding agencies in the public, commercial, or notfor-profit sectors.

\section{References}

1. Lamberti M, Porto S, Marra M, Zappavigna S, Grimaldi A, Feola D, Pesce D, Naviglio S, Spina A, Sannolo N, Caraglia M, 5-Fluorouracil induces apoptosis in rat cardiocytes through intracellular 
oxidative stress. J Exp Clin Cancer Res 2012; 31:60.

2. Sara JD, Kaur J, Khodadadi R, Rehman M, Lobo R, Chakrabarti S, Herrmann J, Lerman A, Grothey A, 5-fluorouracil and cardiotoxicity: a review. Ther Adv Med Oncol 2018; 10: 1-18.

3. Wei Bi, Yue Bi, Pengfei Li, Shanshan Hou, Xin Yan, Connor Hensley, Catherine E. Bammert, Yanrong Zhang, K. Michael Gibson, Jingfang Ju, Lanrong Bi, Indole Alkaloid Derivative B, a Novel Bifunctional Agent That Mitigates 5-Fluorouracil-Induced Cardiotoxicity. ACS Omega 2018; 3: 15850-15864.

4. Murray PJ, The JAK-STAT signaling pathway: input and output integration. J Immunol 2007; 1,178(5):2623-9.

5. Chang H, Zhao F, Xie X, Liao Y, Song Y, Liu C, Wu Y, Wang Y, Liu D, Wang Y, Zou J, Qi Z, PPARa suppresses Th17 cell differentiation through IL-6/STAT3/RORyt pathway in experimental autoimmune myocarditis. Exp Cell Res 2019; 375(1):22-30.

6. Shati AA, El-Kott AF, Acylated ghrelin prevents doxorubicin-induced cardiac intrinsic cell death and fibrosis in rats by restoring IL-6/JAK2/STAT3 signaling pathway and inhibition of STAT1. Naunyn Schmiedebergs Arch Pharmacol 2019; 392(9):1151-1168.

7. Heinrich PC, Behrmann I, Haan S, Hermanns HM, Müller-Newen G, Schaper F, Principles of interleukin (IL)-6-type cytokine signalling and its regulation. Biochem J 2003; 15,374(Pt 1):1-20.

8. Harrison DA, The Jak/STAT pathway. Cold Spring Harb Perspect Biol 2012; 4(3): a011205.

9. Kersten S, Integrated physiology and systems biology of PPARa. Mol. Metab 2014; 3: 354-371.

10. Refaie MMM, Upregulation of peroxisome proliferator activated receptor alpha by fenofibrate in induced testicular ischemia reperfusion. Biomed Pharmacother 2018; 98:507-515.

11. Al-Asmari AK, Khan AQ, Al-Masri N, Mitigation of 5-fluorouracil-induced liver damage in rats by vitamin C via targeting redox-sensitive transcription factors. Hum Exp Toxicol 2016; 35(11):12031213.

12. Mohamed E T, Safwat G M, Evaluation of cardioprotective activity of Lepidium sativum seed powder in albino rats treated with 5-fluorouracil. Beni - suef university journal of basic and applied sciences 2016; 5: 208-215.

13. Rivera-Meza M, Muñoz D, Jerez E, Quintanilla M E, Salinas-Luypaert C, Fernandez K, Karahanian E, Fenofibrate administration reduces alcohol and saccharin intake in rats: possible effects at peripheral and central levels. Front Behav Neurosci 2017; 11: 133.

14. Sun B, Xie Y, Jiang J, Wang Y, Xu X, Zhao C, Huang F, Pleiotropic effects of fenofibrate therapy on rats with hypertriglycemia. Lipids Health Dis2015; 14: 27.

15. Buege JA, Aust SD, Microsomal lipid peroxidation. Methods in enzymology 1978; 52:302-10.

16. Bancroft JD, Gamble M, Theory and practice of histological techniques (Elsevier Health Sciences) 2008; 6th Edition.

17. Ibrahim MA, Geddawy A, Abdel-Wahab S, Sitagliptin prevents isoproterenol-induced myocardial infarction in rats by modulating nitric oxide synthase enzymes. European journal of pharmacology 2018; 829: 63-69. 
18. Côté S, Current protocol for light microscopy immunocytochemistry. Immunohistochemistry 1993; II:148-167.

19. VanGuilder HD, Vrana KE, Freeman WM, Twenty-five years of quantitative PCR for gene expression analysis. Biotechniques 2008; 44: 619-626.

20. Ewees MG, Messiha BAS, Abdel-Bakky MS, Bayoumi AMA, Abo-Saif AA, Tempol, a superoxide dismutase mimetic agent, reduces cisplatin-induced nephrotoxicity in rats. Drug Chem Toxicol 2018; $1-8$.

21. Gelen V, Şengül E, Yıldırım S, Atila G, The protective effects of naringin against 5-fluorouracil-induced hepatotoxicity and nephrotoxicity in rats. Iran J Basic Med Sci 2018; 21(4):404-410.

22. Desai A, Mohammed T, Patel KN, Almnajam M, Kim AS, 5-Fluorouracil Rechallenge After Cardiotoxicity. Am J Case Rep 2020; 21:e924446.

23. Durak I, Karaayvaz M, Kavutcu M, Cimen MY, Kacmaz M, Buyukkocak S, et al, Reduced antioxidant defense capacity in myocardial tissue from guinea pigs treated with 5 -fluorouracil. $J$ Toxicol Environ Health A 2000; 59:585-589.

24. Eskandari MR, Moghaddam F, Shahraki J, Pourahmad J, A comparison of cardiomyocyte cytotoxic mechanisms for 5-fluorouracil and its pro-drug capecitabine. Xenobiotica 2014;1-9.

25. Aikemu A, Amat N, Yusup A, Shan L, Qi X, Upur H,Attenuation effect of Abnormal Savda Munziq on liver and heart toxicity caused by chemotherapy in mice. Exp Ther Med 2016; 12(1):384-390.

26. Helmy MM, Helmy MW, El-Mas MM, Additive renoprotection by pioglitazone and fenofibrate against inflammatory, oxidative and apoptotic manifestations of cisplatin nephrotoxicity: modulation by PPARs. PLoS One 2015; 10(11):e0142303.

27. Rashid S, Ali N, Nafees S, Hasan SK, Sultana S, Mitigation of 5-Fluorouracil induced renal toxicity by chrysin via targeting oxidative stress and apoptosis in wistar rats. Food Chem Toxicol 2014; 66:185193.

28. Refaie MMM, Shehata S, El-Hussieny M, Abdelraheem WM, Bayoumi AMA, Role of ATP-Sensitive Potassium Channel (KATP) and eNOS in Mediating the Protective Effect of Nicorandil in Cyclophosphamide-Induced Cardiotoxicity. Cardiovasc Toxicol 2020; 20 (1):71-81.

29. Arab HH, Salama SA, Maghrabi IA, Camel Milk Ameliorates 5-Fluorouracil-Induced Renal Injury in Rats: Targeting MAPKs, NF-KB and PI3K/Akt/eNOS Pathways. Cell Physiol Biochem 2018; 46(4):1628-1642.

30. Muhammad RN, Sallam N, El-Abhar HS, Activated ROCK/Akt/eNOS and ET-1/ERK pathways in 5fluorouracil-induced cardiotoxicity: modulation by simvastatin. Sci Rep 2020; 10(1):14693.

31. Krysiak R, Gilowski W, Szkrobka W, Okopien B, Different effects of fenofibrate on metabolic and cardiovascular risk factors in mixed dyslipidemic women with normal thyroid function and subclinical hypothyroidism. Cardiovasc Ther 2014; 32(6):264-9.

32. Jen HL, Yin WH, Chen JW, Lin SJ, Endothelin-1-Induced Cell Hypertrophy in Cardiomyocytes is Improved by Fenofibrate: Possible Roles of Adiponectin. J Atheroscler Thromb 2017; 24(5):508-517. 
33. Khan V, Sharma S, Bhandari U, Sharma N, Rishi V, Haque SE, Suppression of isoproterenol-induced cardiotoxicity in rats by raspberry ketone via activation of peroxisome proliferator activated receptoralpha. Eur J Pharmacol 2019; 5(842):157-166.

34. Wang W, Fang Q, Zhang Z, Wang D, Wu L, Wang Y, PPAR alpha Ameliorates Doxorubicin-Induced Cardiotoxicity by Reducing Mitochondria-Dependent Apoptosis via Regulating MEOX1. Front Pharmacol 2020; 11:528267.

35. Huang WP, Yin WH, Chen JS, Huang PH, Chen JW, Lin SJ, Fenofibrate attenuates doxorubicin-induced cardiac dysfunction in mice via activating the eNOS/EPC pathway. Sci Rep 2021;11(1):1159.

\section{Figures}



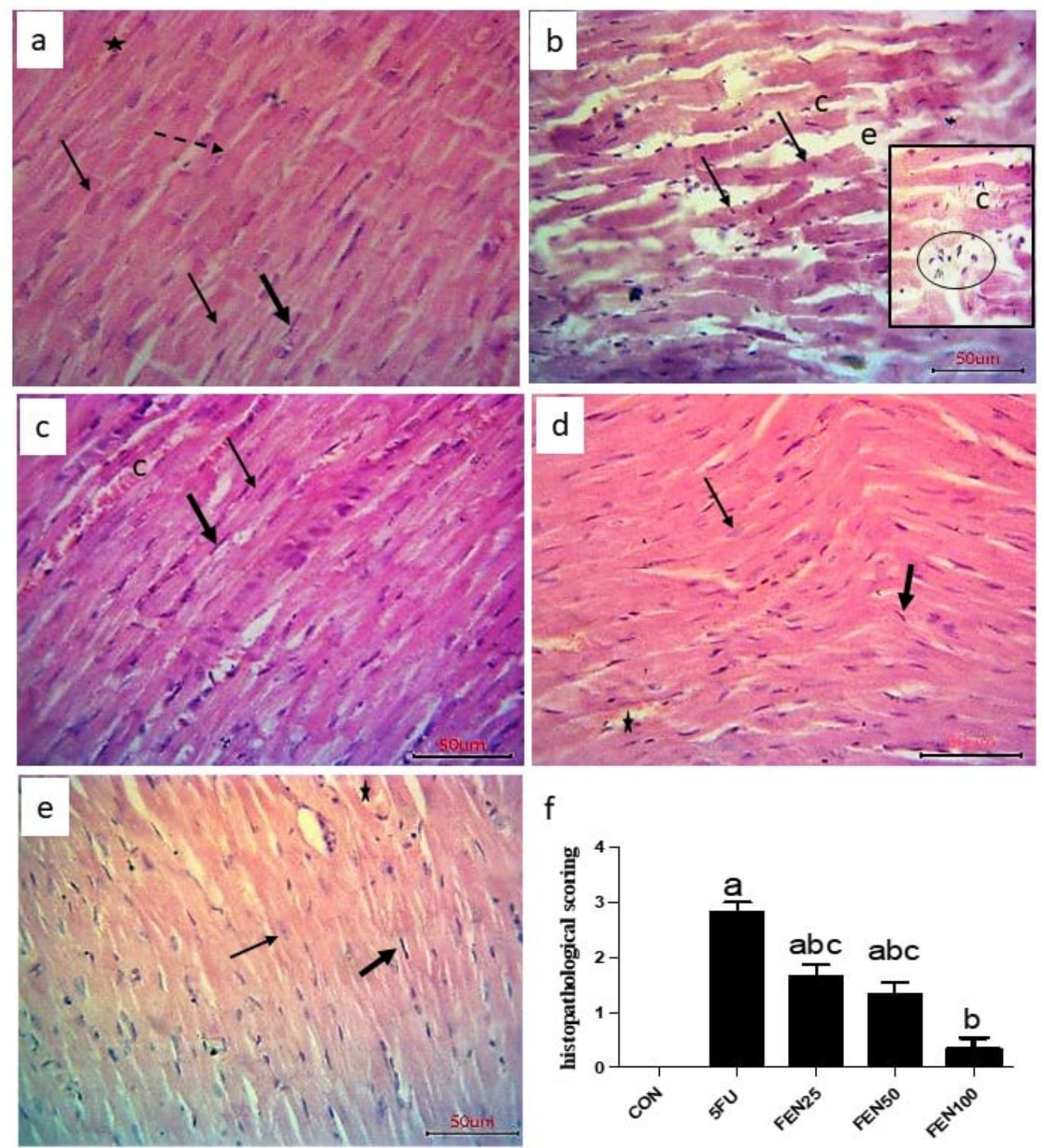

\section{Figure 1}

Photomicrographs of longitudinal section of cardiac muscles a) The control group showing longitudinally arranged cardiac muscle fibers with striated acidophilic cytoplasm and central vesicular oval nuclei (arrows). Notice branching and anastomose of cardiac fibers (dashed arrows), connective tissue cells with their dense flattened nuclei (thick arrows) and the blood capillaries (star) between fibers.

b) 5FU group (5-flurouracil) showing some hyper-eosinophilic fibers with pyknotic nuclei and loss of 
striation (arrows), interstitial oedema (e), marked congestion (c, inset) and inflammatory cells infiltration (circle, inset). c) FEN(fenofibrate) 25treated group, d) FEN (fenofibrate) 50treated group and e) FEN(fenofibrate) 100 treated group showing marked improvement of the myocardial structure; most of myocytes and their nuclei are more or less as the control group with restoration of their transverse striations, less vascular congestion (c) and no inflammatory cell infiltration. f) The histopathogical scoring of myocardial injury. a significantly different from $\mathrm{CON}$ (control), b significantly different from $5 F U$ (5-flurouracil) and c significantly different from FEN(fenofibrate)100-treated group at $p<0.05$ using one-way ANOVA followed by Tukey-Kramer post hoc test. H\&E X400 

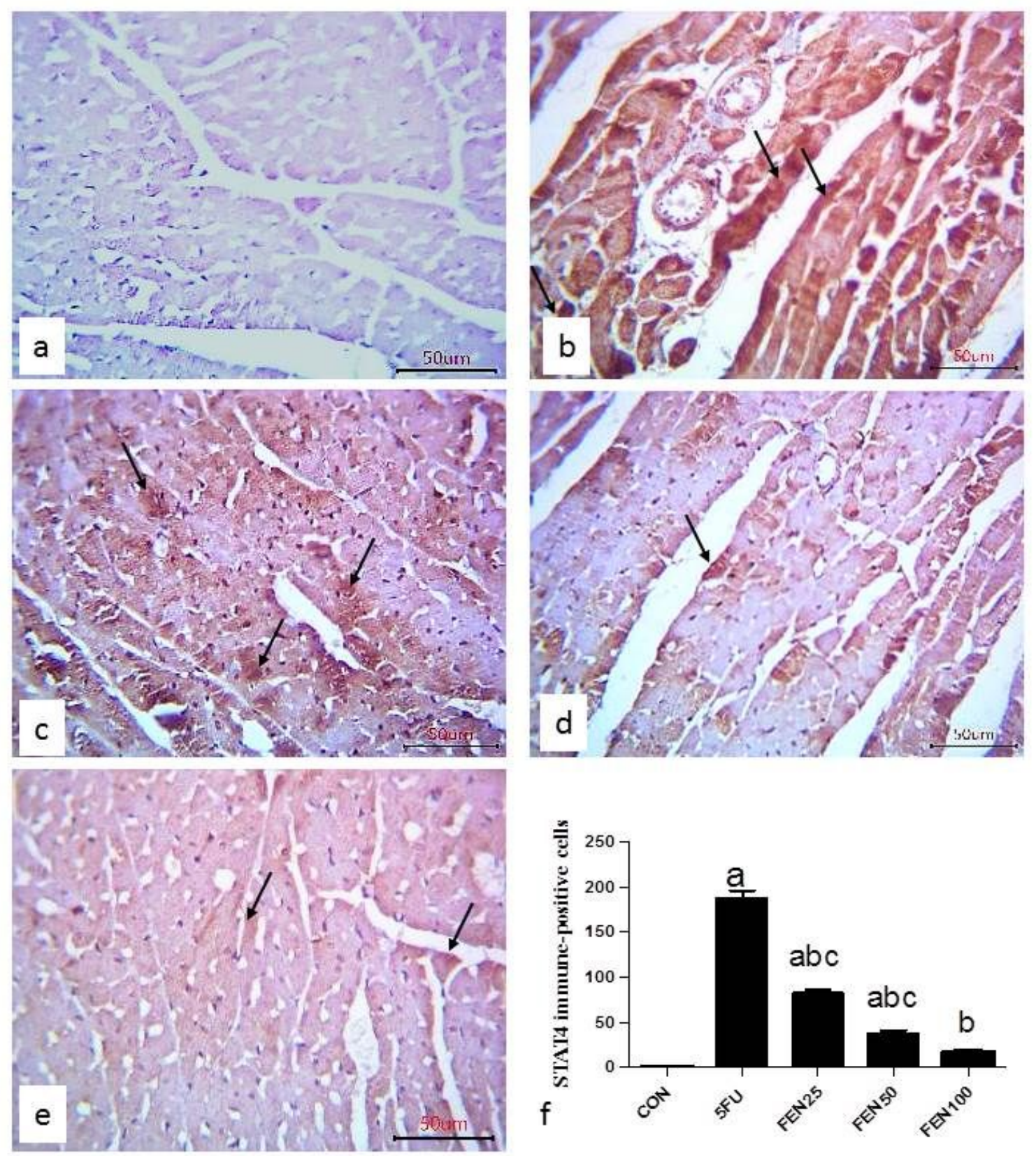

Figure 2

Photomicrographs of transverse sections of cardiac muscles STAT4 immunohistochemical staining: a) CON (control) group showing no immunostaining of cardiac muscle fibers. b) 5FU (5-flurouracil) group showing extensive expression of STAT4 in cardiac muscle fibers (arrows). c) FEN (fenofibrate) 25-treated group, d) FEN (fenofibrate) 50-treated group and e) FEN (fenofibrate) 100-treated group showing markedly decreased expression of STAT4 in cardiac muscle fibers (arrows). f)The mean number of 
STAT4 immune-positive cells in the studied groups expressed as mean \pm SEM $(n=8)$. a Significant $(P$ $<0.05)$ difference from control group. b Significant $(P<0.05)$ difference from $5 F U$ (5-flurouracil) group. $c$ Significant $(P<0.05)$ difference from FEN(fenofibrate) 100 . Immunohistochemical staining for STAT4 X400

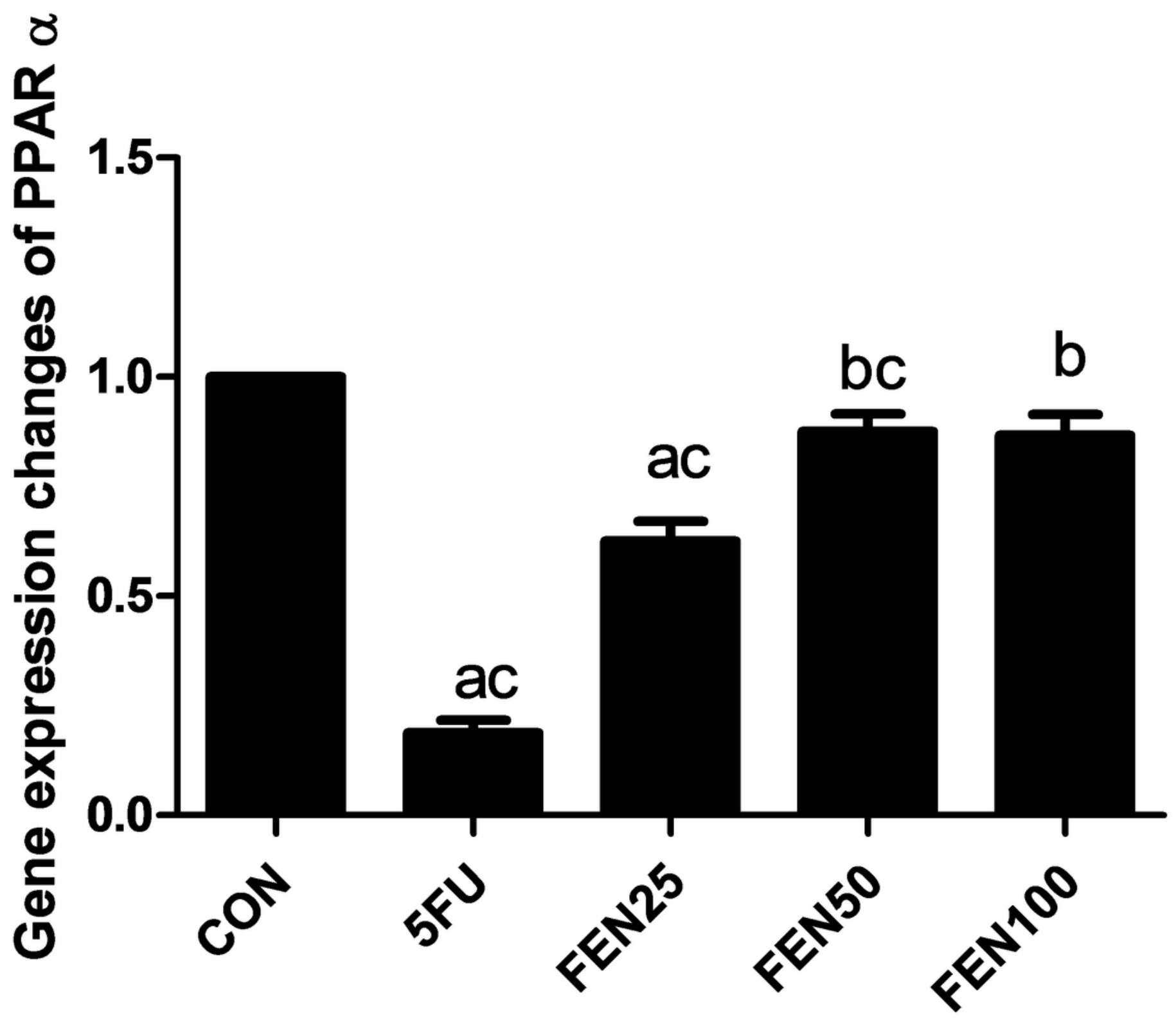

Figure 3

detection of PPARa gene expression changes There is significant decrease of PPARa gene expression in 5FU (5-flurouracil) group in comparison with normal control group. 5FU+FEN (5-flurouracil +fenofibrate) groups showed significant elevation of its gene expression in comparison with 5FU (5-flurouracil) given group alone. 

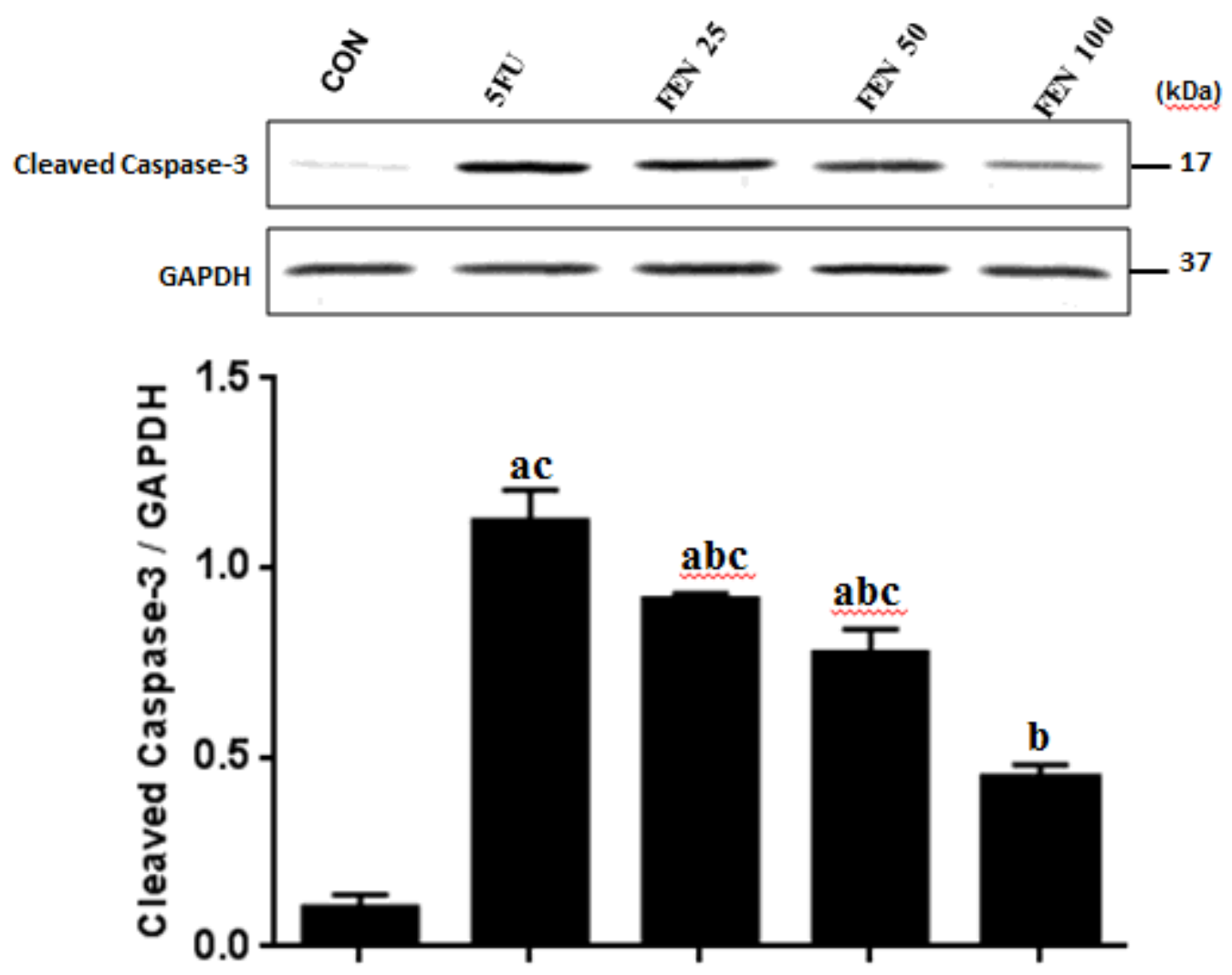

\section{Figure 4}

Detection of interleukin 6 and cleaved caspase 3 by western blotting There is a significant elevation of interleukin 6 and cleaved caspase3 levels in 5-flurouracil group in comparison with normal control group. However, there is a significant decrease of interleukin 6 and caspase 3 levels in fenofibrate groups compared to 5 -flurouracil group. Results represent the mean \pm SEM $(n=8)$. a Significant $(P<0.05)$ difference from control group. b Significant $(P<0.05)$ difference from $5 F U$ (5-flurouracil) group. $C$ Significant $(P<0.05)$ difference from FEN(fenofibrate) 100. 

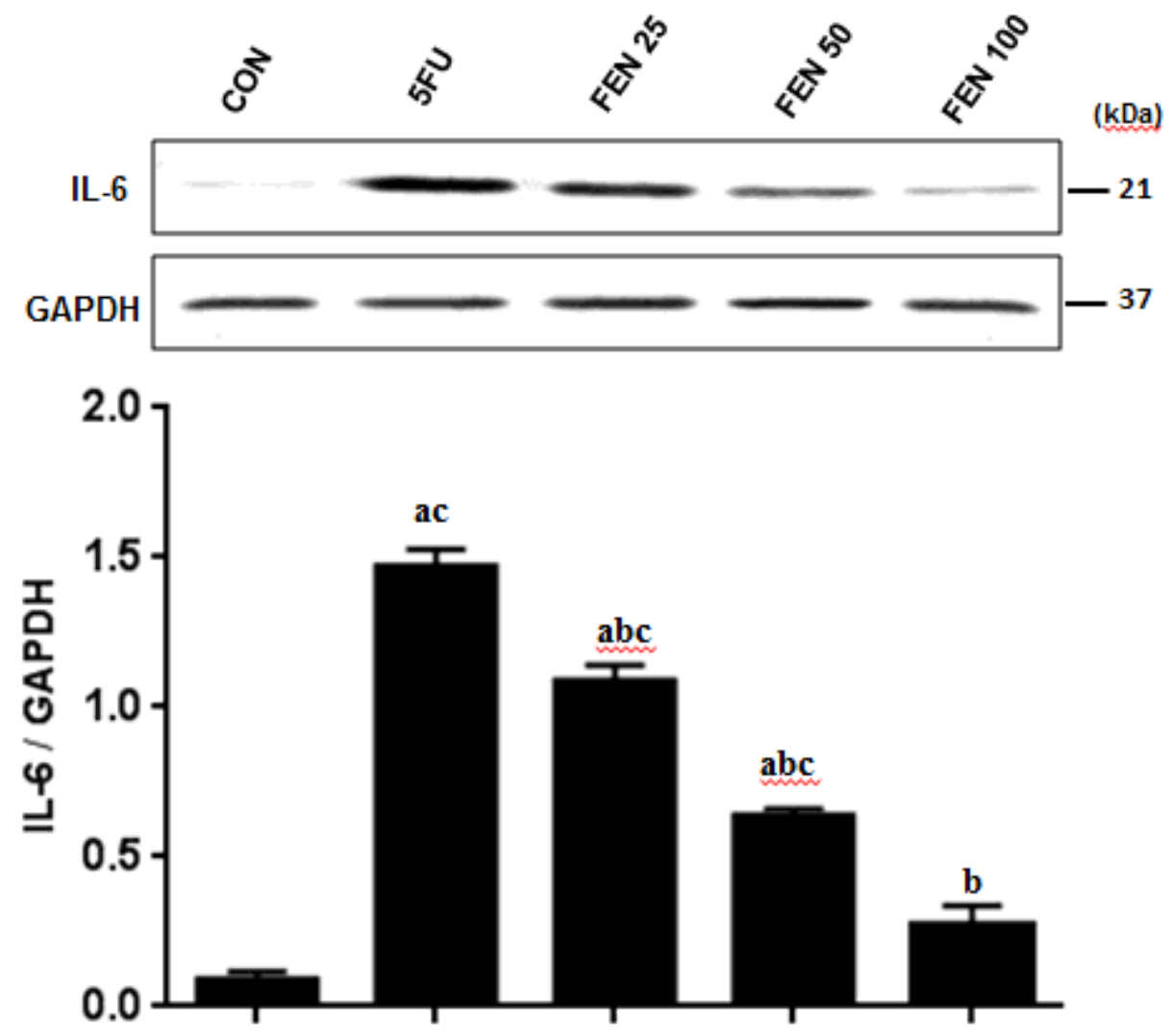

\section{Figure 5}

Detection of interleukin 6 and cleaved caspase 3 by western blotting There is a significant elevation of interleukin 6 and cleaved caspase3 levels in 5-flurouracil group in comparison with normal control group. However, there is a significant decrease of interleukin 6 and caspase 3 levels in fenofibrate groups compared to 5 -flurouracil group. Results represent the mean \pm SEM $(n=8)$. a Significant $(P<0.05)$ difference from control group. b Significant $(P<0.05)$ difference from $5 F U$ (5-flurouracil) group. $C$ Significant $(P<0.05)$ difference from FEN(fenofibrate) 100. 